\title{
Geoeffectiveness of CMEs in the Solar Wind
}

\author{
E. Huttunen \\ University of Helsinki, Finland
}

\begin{abstract}
The main drivers of strong geomagnetic activity at the Earth are interplanetary manifestations of coronal mass ejections. A magnetic storm can be caused by compressed sheath fields before the CME, by the CME ejecta or by the combination of these two structures. The most geoeffective subset of CMEs are magnetic clouds. When observed near 1 AU magnetic clouds are characterized by monotonous rotation of magnetic field direction through a large angle, high magnetic field magnitude, low temperature and low plasma beta. We have investigated the magnetic structure and the geomagnetic consequences of magnetic clouds identified from WIND and ACE data for the years 1997-2003. The geomagnetic response of a certain magnetic cloud depends greatly on its magnetic structure and orientation of sheath fields. We have investigated drivers of intense magnetic storms (Dst $;-100 \mathrm{nT}$ ) during the interval of 1997-2002, i.e. rising, maximum and early declining phases of solar cycle 23 . Sheath regions and post-shock streams caused nearly half of all intense storms. Importance of sheath regions as storm drivers even increased as the level of the storm increased. In 2003 two most intense geomagnetic storms of the solar cycle 23 took place. Both of these were driven by southward fields embedded in a magnetic cloud that had axis highly inclined to the ecliptic plane. Though sheath regions alone efficiently drive intense Dst storms ( $i-100 \mathrm{nT}$ ) the largest storms (Dst $;-300 \mathrm{nT}$ ) require exceptionally long-time and intense southward magnetic fields that presumably only magnetic clouds can provide. High solar wind dynamic pressure seems to be important in generating extremely intense Dst storms. As an example we show solar wind condition during Nov 19-20, 2003 magnetic cloud that caused the largest storm of the solar cycle 23 .

Magnetic clouds have smoothly changing magnetic field direction combined with low solar wind dynamic pressure. Sheath regions typically have rapidly varying magnetic field direction and high dynamic pressure. Thus, these two solar wind drivers put magnetosphere under different type of driving. We also studied the responses of the Dst index that aims to measure the strength of the equatorial ring current and the Kp index that records more global and higher latitude activity than Dst to different storm drivers. We found that in general sheath regions generate higher Kp activity when compared to the level of the the Dst disturbance than magnetic clouds. In some cases rapidly fluctuating magnetic field in the sheath region caused very strong highlatitude activity (Kp 8-9) though the Dst index was significantly less enhanced. This suggest that magnetospheric current systems have different responses to different solar wind drivers.
\end{abstract}

Keywords. Sun: coronal mass ejections (CMEs)

\section{Discussion}

DERE: Did you also consider geomagnetic storms that were not associated with MCs.

Huttunen: The results were shown from two different studies: 1)magnetic clouds from 1997 to 2003 ; 2)drivers of all storms with Dst $<-100 \mathrm{nT}$ from 1997-2002. In 2) we considered all drivers of storms, $25 \%$ of 53 storms were not associated to MCs or sheath regions/shocks. They were driven by ICMEs without flux rope structure or solar wind structures not related to CMEs (e,g., compression regions of slow and high (solar wind streams) speed). 
JIE ZHANG: You attribute storms either to shock sheath or to MC. But in some cases, it must be a combinedl effect. For example, an ICME has both shock and sheath. How do you distinguish when this occurs?

Huttunen: The storm period was determined according to Kamide etal.1998 (whether one storm with two steps or two different storm). The driver of the storm was defined as a feature causing $85 \%$ of the Dst minimum for that storm. We approximated the time when the front edge of a magnetic cloud reached the magnetopause and added a hour to that time to define the time when the effect of the magnetic cloud started. Only in small subset of cases it was difficult to separate the effect of sheath and magnetic cloud (for SN type magnetic clouds) 